\title{
La investigación científica en los estudiantes de la UNAH
}

PORTAL DE LA CIENCIA (PC) Entrevista a Jorge Danilo López Pavón. Es un investigador de la ciudad de Tegucigalpa. Realizó estudios de licenciatura en Administración de Empresas y Maestría en Metodologías de Investigación Económica y Social, en la UNAH, cuenta con más de 14 años de experiencia en el sector de la microfinanzas en Honduras y Guatemala; consultor para la UNAH y FLASCO Costa Rica.

$\mathrm{PCl}$ ¿Cómo valora la experiencia de la publicación de investigaciones realizadas en grado y posgrado de la UNAH?

JDLPI Indiscutiblemente el tema de investigación a nivel universitario tiene como objetivo la búsqueda del conocimiento. El tener la oportunidad de compartir los trabajos que a nivel individual se realizan, con el fin de demostrar que los fenómenos sociales son sujetos de estudio de manera permanente. Es una grata experiencia culminar con la publicación de su trabajo. Por consiguiente, la divulgación debe promover y motivar a la población estudiantil a enfrentar los desafíos de la investigación científica.

$\mathrm{PC} /$ ¿Considera usted que los trabajos de investigación de grado pueden ser publicables? ¿De qué depende?

JDLP/ Cada trabajo debe reunir los estándares requeridos por la academia, especialmente en la rigurosidad metodológica. Es importante tener la claridad en el compromiso asumido en cada investigación, conscientes de que estamos construyendo y compartiendo conocimiento, además de contribuir a encontrar las alternativas de solución de los problemas socioeconómicos en que estamos inmersos.

$\mathrm{PC} /$ ¿Considera usted que los trabajos de investigación de posgrado pueden ser publicables? ¿Qué tipo de trabajos?

JDLP/Erica Lara, en su trabajo sobre Los fundamentos de investigación (2011:139), se refiere a lo mencionado por Longo y Rodeiro, "La comunicación, propuesta por un saber científico disciplinar, conceptualiza el texto académico. Así mismo, éste, utiliza los medios discursivos de la exposición, la explicación y la argumentación. A la vez, se caracteriza en su uso y circulación en las instituciones educativas". Partiendo de esta proposición, podemos considerar que todo trabajo realizado por 
estudiantes o egresados de postgrado, como ser: proyectos científicos, monografías, ensayos, informes, y cualquier otro género académico escrito, apegado a los estándares de rigurosidad de estructura, recursos y lenguaje, puede ser considerado para publicación. Un contenido de alta calidad puede generar oportunidades de reconocimientos, becas, nuevos trabajos, contratos y, lo más importante, que sea utilizada a corto o mediano plazo.

\section{$\mathrm{PCl}$ ¿Qué nivel de exigencia deben aplicar los docentes para que los trabajos de investigación de los estudiantes sean publicables?}

JDLP/ Los docentes deben supervisar que los proyectos de investigación se apeguen a los requerimientos exigidos por los métodos y técnicas de Investigación, apoyados en los términos de referencia de las diferentes revistas científicas con estándares nacionales e internacionales; por otra parte, deberán transmitir sus propios métodos, estilos y su experiencia como investigador, no como un recetario, por el contrario, ser facilitadores para despertar el interés del estudiante universitario para investigar y publicar. Robert A. Day, en su texto Cómo escribir y publicar trabajos científicos, tiene presente lo mencionado por el físicomatemático, Hermann Bondi, sobre la importancia de un buen trabajo científico, quien, señala textualmente que: "La ciencia es una actividad fundamental en la sociedad, lo que implica, que obedece a una excelente comunicación". Por ejemplo, en el ámbito económico podemos encontrar, guías de estilo, en revistas de reconocido prestigio internacional, como American Economic Review (AER), Econométrica, Quartely Journal of Economic (QJE), Journal of Economic Theory (JET), Review of Economic Studies (RES) entre otras. Por consiguiente, los docentes deben tener presente que hoy vivimos bajo un orden globalizado y dinámico, con información expedita y con alta tecnología al alcance de todo aspirante a investigador. En consecuencia, desarrollar competencias en el estudiante orientadas a investigar, debe ser parte fundamental en el proceso de enseñanza-aprendizaje.

\section{$\mathrm{PCl}$ ¿Cuáles son los requisitos mínimos que debe poseer una investigación a nivel de grado y posgrado?}

JDLP/ Hay muchos autores que sugieren aspectos que debe reunir un proyecto de investigación científica. Para responder consideraremos de guía la sugerida por la Universidad Mesoamericana de México:

Puntos básicos del proyecto de investigación 
- Presentación General del Proyecto de Investigación (resumen)

- Título y subtítulo de la investigación

- Objeto de estudio específico

- Estado del arte (¿qué se ha hecho/dicho sobre este objeto de investigación?)

- Ámbitos temáticos de los cuales se va a obtener información general sobre el objeto de estudio específico

- Tópico o tema general al que pertenece la investigación

- Problema práctico: a) condición del problema, b) costo del problema

- Problema de investigación: a) condición y problema, b) costo

- Justificación: a) ¿Por qué es importante hacer la investigación?, b) ¿Qué le sucede a la sociedad si yo no la llevo a cabo?

- Preguntas de investigación:a) Nucleares y b) Periféricas

- Objetivos de investigación: a) Nucleares y b) Periféricos

- Marco teórico interpretativo: a) Nombre de la teoría (s), b) Datos biográficos del autor de la teoría, c) Contexto histórico de surgimiento y desarrollo de la teoría, d) Explicación de la teoría, e)Aplicación de la teoría al objeto de estudio.

- Principales conceptos teóricos

- Hipótesis o conjeturas

- Tipo de investigación: a) por la naturaleza del objeto de estudio, b) por sus objetivos, c) por la temporalidad, d) por la estrategia metodológica, e) por la profundidad de los conocimientos buscados.

- Estrategia metodológica: ¿Cómo se va a llevar a cabo la investigación?

- Técnicas de recopilación de la información: a), b), c),

- Técnicas de análisis de la información:a), b), c)

- Resultados deseados: (lo ideal):

- Impacto esperado: (lo posible):

- Recursos humanos y materiales necesarios para llevar a cabo la investigación

- Cronograma de actividades:

- Índice tentativo/orientativo del reporte escrito de investigación:

- Formatos y estrategias de comunicación y presentación de los resultados.

- Lista de referencias de los documentos recopilados durante la elaboración de proyecto

\section{$\mathrm{PCl}$ ¿Qué acciones podrían realizarse para lograr entre los estudiantes de posgrado el empoderamiento del proceso investigativo?}

JDLPI Invitarle a ser parte de equipos interdisciplinarios de investigación científica,conformando un bloque de investigadores auxiliares con investigadores experimentados. Por consiguiente, se garantizaría el crecimiento y desarrollo individual, con incidencia en cualquiera de los ejes de investigación de las áreas programáticas académicas de la UNAH. 


\section{$\mathrm{PCl} ¿ Q$ ué hacer para promover la investigación en los estudiantes de grado y posgrado? ¿Qué papel juegan los profesores e investigadores?}

JDLP/ Hacer extensiva la investigación con variedad de incentivos que impulsen la competitividad entre facultades, con temas nacionales; así mismo, que mediante la vinculación universitaria estas investigaciones se relacionen con los problemas reales de la población. Esa será la responsabilidad de orientar al estudiante por parte de los profesores e investigadores.

\section{$\mathrm{PCl}$ ¿Cómo promover una cultura de publicación en los estudiantes?}

JDLPI Una de las principales preocupaciones de la oficina regional de la UNESCO para América Latina y El Caribe, es la falta de interés y el rechazo al estudio de las ciencias, por parte de los jóvenes en educación secundaria y, en consecuencia, la falta de candidatos para estudios científicos en el nivel educativo superior. Una de sus recomendaciones es retomar "la alfabetización científica" que consiste en involucrar a la comunidad estudiantil en las tomas de decisiones en nuestro diario vivir, desarrollar capacidades de discusión pública y académica, acerca de asuntos relacionados con la ciencia y la tecnología. Es imperante que en los sistemas educativos nacionales, e internacionales, especialmente en el ámbito estudiantil universitario hondureño, se presenten planteamientos de hacia dónde y cómo avanzar al campo de la investigación e innovaciones educativas, con la finalidad de promover la cultura de publicaciones científicas en los estudiantes universitarios. Por consiguiente el estudiante debe aprender a crear productos científicos de calidad y enfatizar que el paso final, del proceso, es la publicación.

\section{$\mathrm{PC} /$ ¿Cuáles son los retos más grandes que debe enfrentar la universidad para promover y visibilizar más el papel de los estudiantes en las investigaciones que desarrollan los investigadores con más experiencia?}

JDLPI Los países desarrollados han apostado a la importancia de la investigación por ser un espacio formativo y por sus aportes a producir ciencia. Promover y motivar al estudiante universitario representa otro desafío, aún mayor, debido a las falencias en el proceso formativo. La universidad debe transformar, provocar cambios, y los estudiantes deben competir en esta nueva plataforma, con todas las herramientas, para visibilizar su papel con los que desarrollan proyectos de investigación. Representa un reto, de vital importancia, en lo que respecta a la formación de investigadores nacionales, con el compromiso de estudiar y dar respuestas de solución a los diferentes problemas que enfrenta nuestro país. 


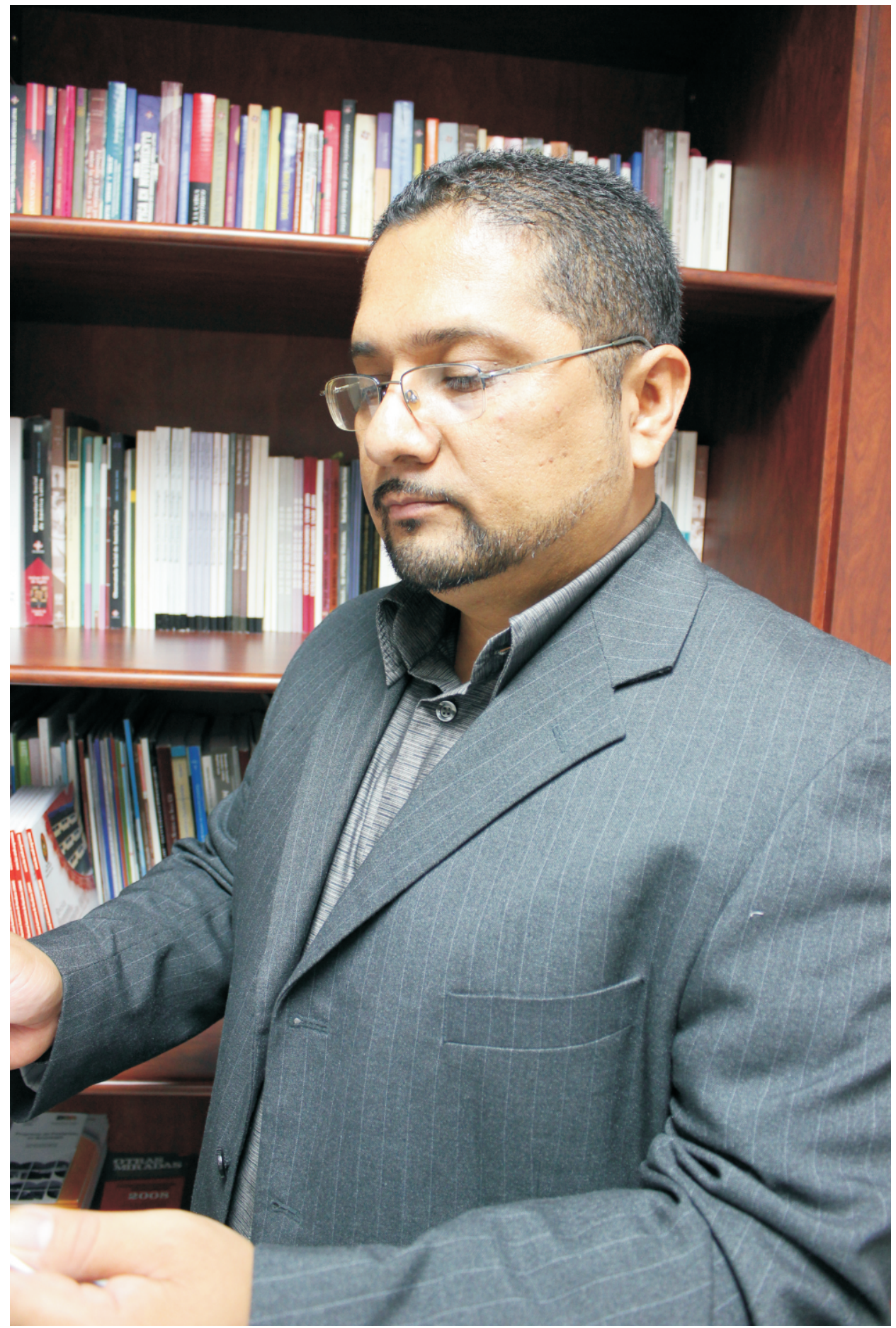


PORTAL DE LA CIENCIA (PC) Entrevista al economista, Gabriel Perdomo, es un joven investigador originario de la ciudad de Tegucigalpa. Realizó estudios de licenciatura en Economía y la Maestría en Metodologías de Investigación Económica y Social en la Universidad Nacional Autónoma de Honduras UNAH.

Actualmente labora en el Foro Social de la Deuda Externa y Desarrollo de Honduras, FOSDEH, como investigador y analista, realizando trabajos de lobby e incidencia con actores clave con énfasis en políticas fiscales y de desarrollo.

\section{PCl ¿Cómo valora la experiencia de la publicación de investigaciones realizadas en grado y posgrado de la UNAH?}

GP/ Las publicaciones científicas han experimentado un aumento considerable, tanto en calidad como en cantidad en los últimos años, por lo cual algunas facultades han obtenido mayor nivel de publicaciones que otras. Pero en los niveles de grado y posgrado, seguimos experimentando al tener una gran cantidad de tesis e investigaciones que solamente han servido con el propósito de aprobar, como requisito para graduarse, pero estos documentos no han sido utilizados en la investigación y desarrollo ante la falta de un sistema fortalecido integral de acceso a información científica nacional con una vinculación entre la academia, gobierno, empresa y sociedad civil. Todavía existe un largo camino por recorrer para establecer en Honduras una comunidad científica de alto nivel con una relación importante en el desarrollo de nuestro país.

\section{$\mathrm{PC} /$ ¿Considera usted que los trabajos de investigación de grado pueden ser publicables? ¿De qué depende?}

GPI A nivel de grado considero que es donde existe una mayor debilidad, ahí lo más importante es el rigor científico impartido y exigido para las investigaciones realizadas en las carreras universitarias. A esta situación se le suma la debilidad institucional de las facultades en promover el emprendimiento científico en sus estudiantes y catedráticos.

Las publicaciones a nivel de grado dependen de la mejora de la calidad de las clases impartidas en metodologías de investigación, armonizando todas las carreras de la universidad para tener científicos jóvenes aplicando un paradigma de investigación, según sea el problema y objetivos de la tesis para darle la solidez a las publicaciones mediante el rigor científico. 


\section{$\mathrm{PCl}$ ¿Considera usted que los trabajos de investigación de posgrado pueden ser publicables? ¿Qué tipo de trabajos?}

GPI Los trabajos de posgrado tienen una mayor justificación para ser publicados, pero esto debe atender varias situaciones negativas que disminuyen la calidad científica de los mismos. Como en el nivel de grado, las investigaciones de posgrado sufren, en muchas ocasiones, de la falta de rigor metodológico, la universidad necesita impulsar con una mayor fortaleza las clases de alto nivel y contenido metodológico para la investigación científica. Sin embargo, aunque se realice una investigación metodológicamente fuerte, no tendrán un impacto considerable si no se vincula la academia con los hacedores de políticas públicas, para que dicha tesis pueda darle el respaldo científico a las acciones del gobierno y las empresas en el amplio marco de alcanzar un estadio mejor de desarrollo socioeconómico y ambiental del país.

\section{$\mathrm{PC} /$ ¿Qué nivel de exigencia deben aplicar los docentes para que los trabajos de investigación de los estudiantes sean publicables?}

GP/ Más que un nivel de exigencia, un mayor nivel de enseñanza por parte de los docentes, donde la exigencia sea el rigor científico que el estudiante aplique en las publicaciones tanto metodológicas, como de la forma efectiva en que elabore un artículo científico en cumplimiento con las exigencias de las revistas científicas internacionales con mayor renombre en las distintas ciencias.

\section{$\mathrm{PCl}$ ¿Cuáles son los requisitos mínimos que debe poseer una investigación a nivel de grado y posgrado?}

GPI En ambos niveles, los requisitos deben de ser inicialmente un paradigma de investigación claro y con un diseño estructural que permita mantener la consistencia y marco lógico desde el planteamiento del problema hasta el diseño de los instrumentos y técnicas de recolección de datos.

El estudiante debe hacerse preguntas epistemológicas en cuanto a si su investigación es verdaderamente ciencia y muestra la realidad de forma fidedigna. Por último, pero no por esto menos importante, es un requisito esencial que en Honduras dentro de la pequeña comunidad científica existe una debilidad y es la elaboración del marco teórico. Este requerimiento es el que necesita ser potencializado, ya que para que un documento se convierta en científico debe ir respaldado con un sustento teórico científico, aunque la investigación sea exploratoria de un problema no estudiado, siempre se debe tener un producto de la 
revisión de la literatura, como un sustento teórico de lo que probablemente sea la realidad del problema de investigación.

Todo este proceso debe de ser finalizado con los "peer reviews". Toda investigación debe de ser compartida y revisada por metodólogos y científicos en el tema para darle un mayor sustento a la misma.

PCl ¿Qué acciones podrían realizarse para lograr entre los estudiantes de grado el empoderamiento del proceso investigativo?

GP/ Es importante generar una cohesión social en el estudiante, en el que éste sienta que su trabajo e investigación realmente ayuda a mejorar el país. Se debe crear una plataforma de masificación mediática de la producción científica de documentos a través de los canales de medios electrónicos, conferencias y mesas de debates para estudiantes y docentes, ferias de ciencias, entre otras; donde todos estos procesos estén entrelazados con los diferentes sectores del Estado, empresa privada y sociedad civil para el uso y puesta en práctica de este conocimiento.

\section{$\mathrm{PCl}$ ¿Qué acciones podrían realizarse para lograr entre los estudiantes de posgrado el empoderamiento del proceso investigativo?}

GPIEn Honduras, como en Estados Unidos, debe empezarse a sustentar cualquier política pública en cualquier campo de la ciencia (salud, educación, infraestructura, seguridad social, etc) con investigaciones que puedan guiar a los gobiernos y la sociedad en los resultados de determinada acción estatal. Es momento en que los científicos puedan exponer la ruta correcta a tomar en un Congreso Nacional, Consejo de Ministros o una Corte de Justicia, donde este científico pueda compartir las herramientas para construir políticas públicas. Esto le dará el empoderamiento necesario para que el estudiante de posgrado genere conocimiento científico de mayor rigor teniendo la conciencia de que éste va a ser utilizado para un construir un mejor futuro.

\section{$\mathrm{PCl}$ ¿Qué hacer para promover la investigación en los estudiantes de grado y posgrado? ¿Qué papel juegan los profesores e investigadores?}

GPI Los profesores deben mirar desde la epistemología sí realmente lo que imparten es ciencia y está siendo utilizada por los estudiantes para generar ciencia. Las debilidades especialmente en las metodologías de investigación atrofian la producción de investigadores en la universidad, por esta razón tenemos tan pocos docentes y estudiantes investigadores. Lo primordial es fortalecer la enseñanza 


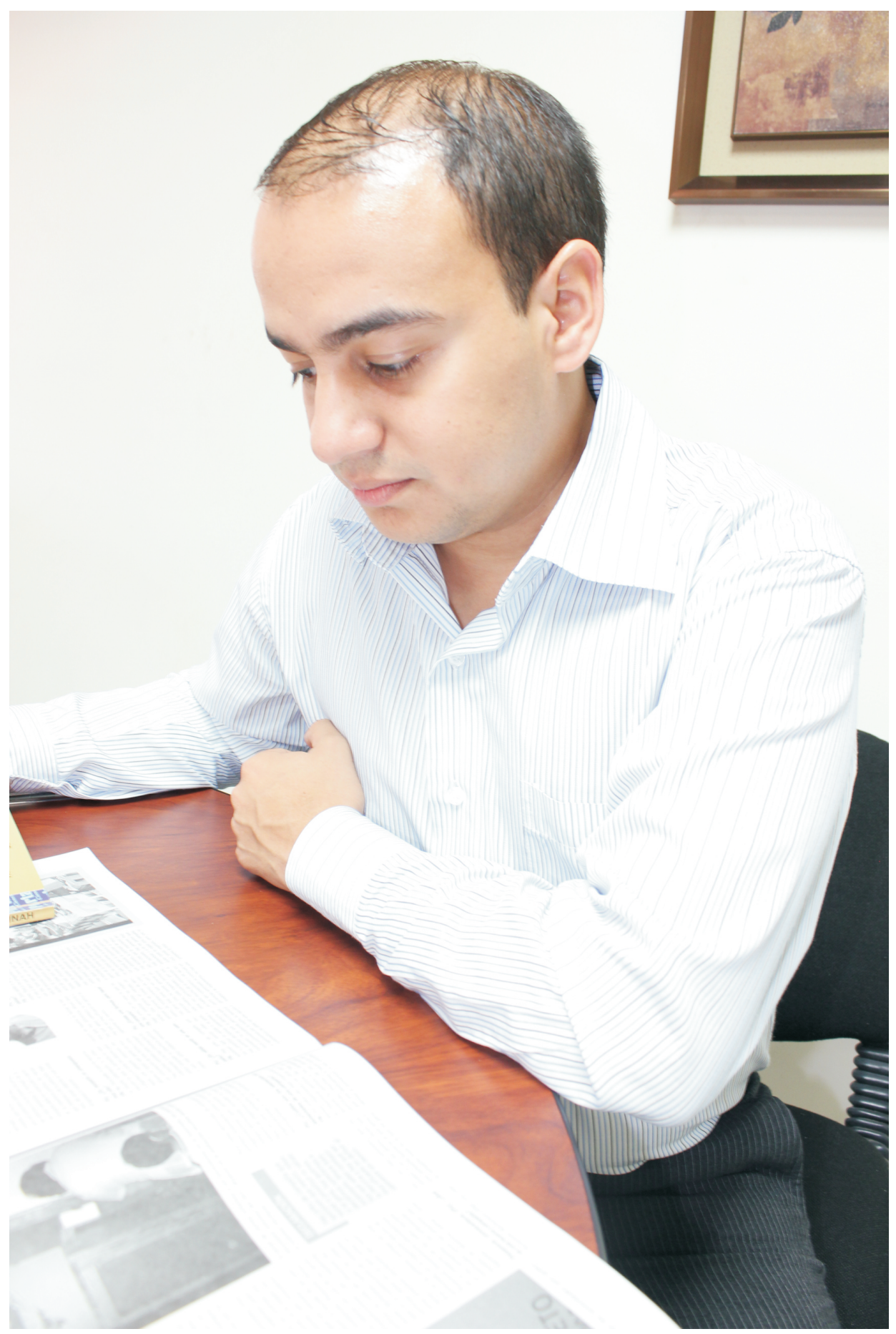


curricular de metodologías de investigación, plataformas y sistemas que permitan un mayor y mejor acceso a todo el conocimiento producido.

\section{$\mathrm{PCl}$ ¿Cómo promover una cultura de publicación en los estudiantes?}

GPI Para promover una cultura, en la que se genere una certeza de publicación se debe promover una cultura de lectura. Para generar una certeza en el estudiante de que su publicación será leída y tomada por diferentes actores en el ámbito del país, inclusive a nivel internacional, mediante las plataformas y sistemas de información científica.

\section{$\mathrm{PCl}$ ¿Cuáles son los retos más grandes que debe enfrentar la universidad para promover y visibilizar más el papel de los estudiantes en las investigaciones que desarrollan los investigadores con más experiencia?}

GPI En 1863, el presidente Abraham Lincoln de los Estados Unidos fundó la Academia Nacional de Ciencias, para que ésta guiara a la nación en los diferentes campos de las ciencias. La Universidad debe convertirse en el bastión del conocimiento científico encaminado al mejoramiento de la sociedad hondureña. Para que las investigaciones producidas por los docentes y estudiantes puedan posicionarse en la esfera pública y tomar el liderazgo científico. Es por esta razón que los retos van encaminados en esta situación, pues hay una debilidad institucional en utilizar y encaminar las investigaciones para el objetivo de supremo de mejorar la calidad de la vida humana. 